\title{
P51 Effects of Pulsatile Exercise-induced Shear Stress on eNOS, SOD, VCAM-1, and ICAM-1 mRNA Expression of Human Carotid Artery Endothelial Cells
}

Francisco Morales-Acuna ${ }^{1, *}$, Alvaro Gurovich

The University of Texas at El Paso, El Paso, USA

\section{ABSTRACT}

Exercise-induced endothelial shear stress (ESS) has been proposed as a molecular mechanism that regulates the expression of genes involved in the protection against atherosclerosis. However, research on this topic has not considered the pulsatile nature of blood flow for in vivo ESS estimations.

Purpose: To analyze the effect of pulsatile exercise-induced ESS on endothelial nitric oxide synthase (eNOS), superoxide dismutase (SOD), vascular cell adhesion molecule 1 (VCAM-1), and intercellular adhesion molecule 1 (ICAM-1) mRNA expression of human carotid artery endothelial cells.

Methods: First, 24 apparently healthy young subjects (14 females and 10 males) performed an exercise test on cycle ergometer at low, moderate, and high intensity in which ESS where estimated from the left common carotid artery through Dopplerultrasound for each condition. Thereafter, commercially available cultured human carotid artery endothelial cells were exposed to pulsatile flow inside a chamber system (Ibidi) for 4 hours, simulating 1 hour of exercise-induced ESS and 3 hours of resting ESS. Finally, eNOS, SOD, VCAM-1, and ICAM-1 mRNA expression were compared between groups, using GAPDH as the housekeeping gene.

Results: ESS showed an intensity-dependent increment to exercise. A significant increment on eNOS mRNA expression $(p<0.05)$ and a significant reduction on SOD mRNA expression $(p<0.05)$ were observed on those cells exposed to moderate and high intensity exercise-induced ESS compared to resting ESS. No significant differences were detected on mRNA expression of VCAM-1 and ICAM-between groups.

Conclusion: Pulsatile ESS generated during moderate and high intensity cycling might favor the upregulation of eNOS and the downregulation of SOD.

(c) 2019 Association for Research into Arterial Structure and Physiology. Publishing services by Atlantis Press International B.V. This is an open access article distributed under the CC BY-NC 4.0 license (http://creativecommons.org/licenses/by-nc/4.0/). 